\title{
Duchenne muscular dystrophy hiPSC- derived myoblast drug screen identifies compounds that ameliorate disease in mdx mice
}

\author{
Congshan Sun, ${ }^{1,2}$ In Young Choi, ${ }^{3}$ Yazmin I. Rovira Gonzalez, ${ }^{2,4}$ Peter Andersen, ${ }^{3,5}$ \\ C. Conover Talbot Jr., ${ }^{6}$ Shama R. Iyer, ${ }^{7}$ Richard M. Lovering, ${ }^{7,8}$ Kathryn R. Wagner, ${ }^{1,2}$ and Gabsang Lee ${ }^{1,3}$
}

'Departments of Neurology and Neuroscience, Johns Hopkins University School of Medicine, Baltimore, Maryland,

USA. Center for Genetic Muscle Disorders, Hugo W. Moser Research Institute at Kennedy Krieger Institute, Baltimore,

Maryland, USA. ${ }^{3}$ Institute for Cell Engineering, ${ }^{4}$ Cellular and Molecular Medicine Graduate Program, and ${ }^{5}$ Division of Cardiology, Department of Medicine, Johns Hopkins University School of Medicine, Baltimore, Maryland, USA. ${ }^{6}$ The Johns Hopkins School of Medicine Institute for Basic Biomedical Sciences, Baltimore, Maryland, USA. ${ }^{7}$ Department of Orthopaedics and ${ }^{8}$ Department of Physiology, University of Maryland School of Medicine, Baltimore, Maryland, USA.

Authorship note: KRW and GL are co-corresponding authors.

Conflict of interest: $\mathrm{CS}, \mathrm{PA}, \mathrm{KRW}$, and GL are founders of Vita Therapeutics. KRW discloses that she is a consultant for Asklepios BioPharmaceutical,

Vita Therapeutics, and Casma

Therapeutics and has received research support from Sarepta Therapeutics, F. Hoffmann-La Roche, Catabasis Pharmaceuticals, Fulcrum Therapeutics, PTC Therapeutics, and Pfizer.

Copyright: (c) 2020, American Society for Clinical Investigation.

Submitted: October 16, 2019

Accepted: April 23, 2020

Published: April 28, 2020.

Reference information: JCI Insight. 2020;5(11):e134287.

https://doi.org/10.1172/jici.

insight.134287.
Duchenne muscular dystrophy (DMD) is the most common muscular dystrophy. In the present study, when human induced pluripotent stem cells (hiPSCs) were differentiated into myoblasts, the myoblasts derived from DMD patient hiPSCs (DMD hiPSC-derived myoblasts) exhibited an identifiable DMD-relevant phenotype: myogenic fusion deficiency. Based on this model, we developed a DMD hiPSC-derived myoblast screening platform employing a high-content imaging (BD Pathway 855) approach to generate parameters describing morphological as well as myogenic marker protein expression. Following treatment of the cells with 1524 compounds from the Johns Hopkins Clinical Compound Library, compounds that enhanced myogenic fusion of DMD hiPSC-derived myoblasts were identified. The final hits were ginsenoside Rd and fenofibrate. Transcriptional profiling revealed that ginsenoside $R d$ is functionally related to FLT3 signaling, while fenofibrate is linked to TCF- $\beta$ signaling. Preclinical tests in $\mathbf{m d x}$ mice showed that treatment with these $\mathbf{2}$ hit compounds can significantly ameliorate some of the skeletal muscle phenotypes caused by dystrophin deficiency, supporting their therapeutic potential. Further study revealed that fenofibrate could inhibit mitochondrion-induced apoptosis in DMD hiPSC-derived cardiomyocytes. We have developed a platform based on DMD hiPSC-derived myoblasts for drug screening and identified 2 promising small molecules with in vivo efficacy.

\section{Introduction}

Duchenne muscular dystrophy (DMD) is an X chromosome-linked disease that affects 1 in 5000 boys worldwide (1). The disease is caused by a mutation in the gene encoding dystrophin, which, along with a glycoprotein complex, connects the intracellular cytoskeleton to the extracellular matrix (2-4). Boys who are born with this disease experience loss of ambulation in early teenage years and die in early adulthood due to cardiac and respiratory complications (2). To this day, treatment options for DMD patients are very limited. Glucocorticosteroid therapy has been the main treatment for almost 3 decades, has limited benefit, and is associated with several significant side effects (5). Exon skipping therapy targets a limited population of DMD patients, and its efficacy remains uncertain $(6,7)$. Meanwhile, gene therapy with adeno-associated virus (AAV) and mini-dystrophin transgene has entered clinical trials, but preexisting immunity to AAV may hinder its availability to large numbers of DMD boys $(8,9)$. Even in the best-case scenario, gene therapy promises to convert a DMD to a milder allelic form of muscular dystrophy, Becker muscular dystrophy phenotype. Therefore, there is an urgent need to search for alternative treatment options for DMD. In order to achieve this, developing an efficient drug screening platform is essential 
To date, for the most part drug preclinical screening and testing have been conducted in DMD animal models, with the $m d x$ and the canine golden retriever muscular dystrophy models being the most commonly employed (10). These models are suitable for drug validation, but not amenable for large-scale screening due to the time-consuming drug development stage and high costs associated (11). As an alternative for largescale drug screening, dystrophin-deficient Caenorhabditis elegans and zebrafish models have been used (12, 13); however, hit compounds found using these organisms have failed to successfully translate into effective DMD treatments (14). While the most promising DMD model for effective drug discovery has relied on use of DMD patient myoblasts, the major restrictions in their application are that myoblasts obtained from DMD patient biopsies are limited in number and phenotypically diverse. In this study, we circumvent the poor expandability of primary myoblasts by using human induced pluripotent stem cells (hiPSCs).

We recently developed a novel system to differentiate DMD hiPSCs into myoblasts using chemically defined conditions that are free of animal feeder cells, serum, or growth factors (15). This myogenic specification protocol involves plating single hiPSCs on defined extracellular matrix material and growing them for 25-30 days in serum-free medium with temporal activation of WNT and inhibition of Notch pathways. On day 25-30, myoblasts can be purified by $\mathrm{NCAM}^{+} / \mathrm{HNK}^{-}$cell surface markers. One reproducible and distinguishable DMD disease phenotype of hiPSC-derived myoblasts is a deficiency in myoblast differentiation and fusion $(15,16)$. Therefore, we designed a high-content imaging-based screening platform to identify compounds that can correct DMD hiPSC-derived myoblast fusion defects. After performing tiered screening with small-molecule compounds from the Johns Hopkins Clinical Compound Library (JHCCL), 2 final hit compounds were selected and further studied to elucidate their mechanism of action, and subsequently tested preclinically in $m d x$ mice and in hiPSC-derived cardiomyocytes (CMs), demonstrating their effectiveness and therapeutic potential. Overall, we performed a comprehensive drug screen using DMD hiPSC-derived myoblasts and demonstrated its feasibility as a platform to identify potential drugs that could be used to treat DMD.

\section{Results}

Primary screening of a small-molecule compound library using DMD patient hiPSC-derived myoblasts. We generated DMD patient hiPSC-derived myoblasts in a chemically defined system of Wnt activation and Notch inhibition from the D2325 hiPSC line of a DMD patient (referred to hereafter as "D2 myoblasts"). The DMD gene in these D2 myoblasts carried a nonsense mutation (c.457C > T) that completely abolished dystrophin protein expression (15) (Supplemental Figure 1, A and B; supplemental material available online with this article; https://doi.org/10.1172/jci.insight.134287DS1). Compared with healthy hiPSC-derived myoblasts, D2 myoblasts formed very few myotubes based on myosin heavy chain (MyHC) antibody staining (Supplemental Figure 1, C and D). This is consistent with our previous studies, in which myoblasts derived from multiple DMD hiPSC lines with various DMD gene mutations formed significantly fewer myotubes, based on MyHC staining (15-17), and similar observations made on primary myoblasts of DMD patients (17-19). Inefficient myotube formation was partially reversed by a known stop codon readthrough compound, gentamicin (Figure 1A) (20). Although not used in the clinical setting due to an unfavorable risk-benefit profile (21), gentamicin served as a positive control in our screen. To test the feasibility of the compound screening format, we imaged myoblasts treated with gentamicin or vehicle control (DMSO) and analyzed them with a high-content imaging analysis system (BD Pathway 855) that could detect and outline the cells on the plate (Figure 1A). We compared a variety of parameters after the treatment of gentamicin or vector DMSO on D2 myoblasts as a pilot experiment in differentiation conditions, and found that when the average length of cells was considered, positive and negative control values were statistically different $\left(Z^{\prime}=0.59\right)$ (Figure $\left.1 C\right)$. MyHC immunofluorescence intensity was also considered, as MyHC protein expression is a hallmark of myotube formation (22). To minimize plate-to-plate variation, we normalized the values obtained from each tested compound to positive and negative controls. Thus, we established algorithm 1 as the following equation: normalized cell average length $+0.3 \times$ normalized MyHC intensity $\left(Z^{\prime}=0.58\right)$ (Figure $\left.1 \mathrm{~B}\right)$. Also, in order to include the compounds that can restore the myotube shape without increasing MyHC immunofluorescence intensity, we used absolute values of cell average length (without normalization) as algorithm 2, which also helped to eliminate the compounds that were autofluorescent.

We seeded D2 myoblasts onto 96-well plates and screened 1524 small-molecule compounds from the compound library, which contained both FDA- and foreign agency-approved drugs (23). The compounds in the library are structurally diverse, and some are natural compounds. Therefore, they are suitable for 


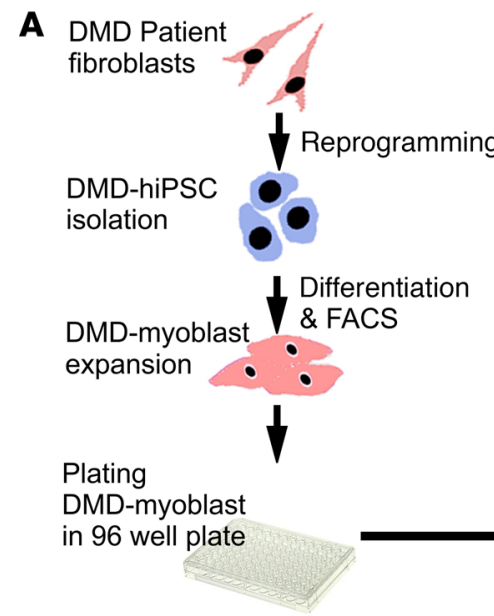

B

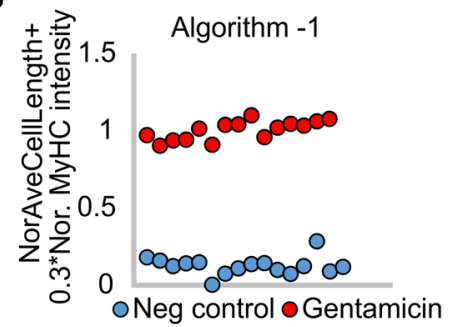

C

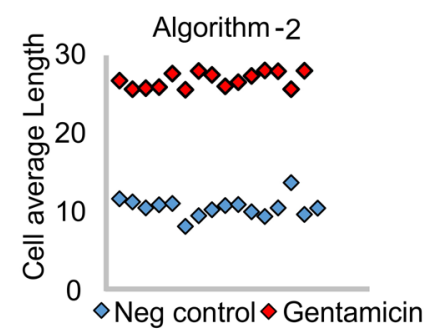

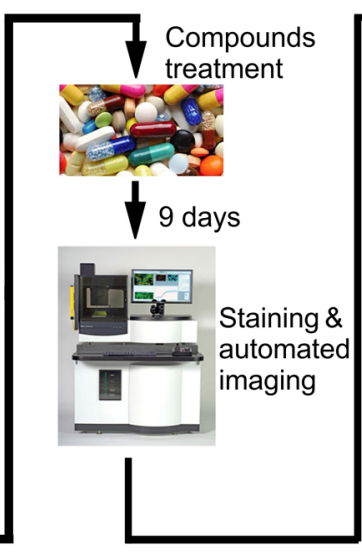

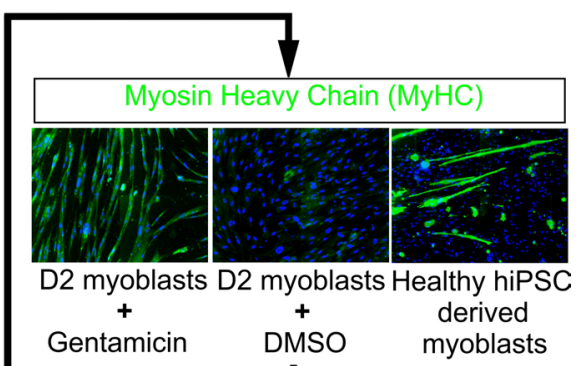

Gentamicin

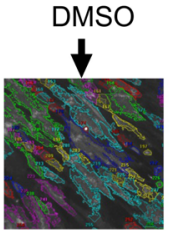

Automated image analysis

\section{D}

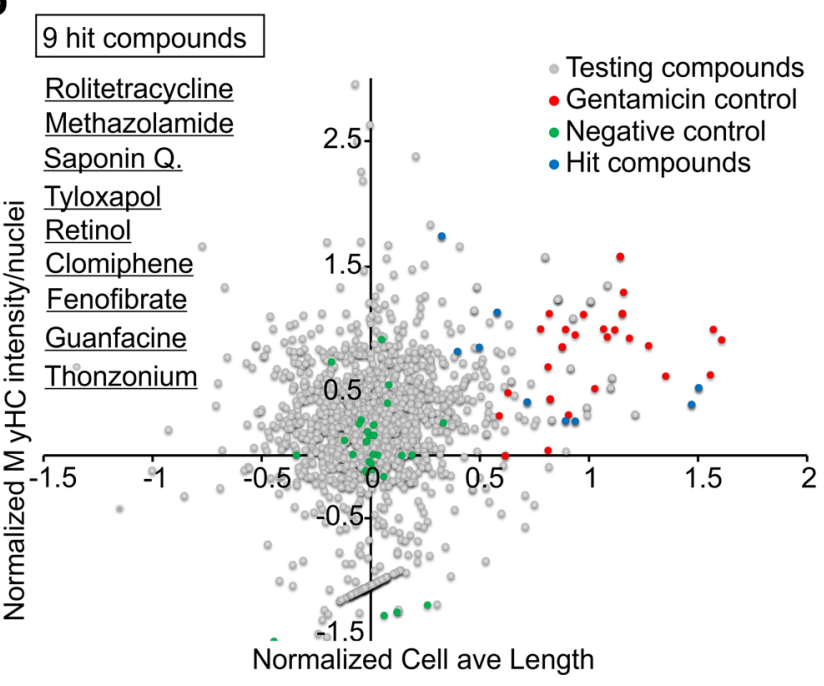

Figure 1. Primary compound screening reveals $\mathbf{9}$ hit compounds. (A) Flow chart of primary compound screening process: Fibroblasts were induced to hiPSCs and differentiated into myoblasts. These patient iPSC-derived myoblasts were expanded and treated with compounds from JHCCL (v1.3). Cells were fixed and stained with MyHC antibody, imaged, and analyzed with a BD Pathway 855 automated imaging system. (B) Graph of distinguishable algorithm 1 values between gentamicin- and DMSO-treated D2 myoblasts, $n$ (negative control) $=16, n$ (gentamicin) $=15\left(Z\right.$ score, $\left.Z^{\prime}=0.58\right)$. NorAveCellLength, normalized cell average length. (C) Graph of distinguishable algorithm 2 values between gentamicin- and DMSO-treated D2 myoblasts, $n$ (negative control) $=16, n$ (gentamicin) $=15$ ( $z$-score, $\left.Z^{\prime}=0.59\right)$. (D) 9 primary hit compounds (listed) were plotted based on 2 parameters used in algorithms: normalized cell average length and normalized MyHC intensity, $n$ (testing compounds) $=1324, n$ (hit compounds) $=9, n$ (negative control) $=19, n$ (gentamicin $)=19$. Each point represents result from 1 well of cells from 96-well plates .

drug repurposing for rare diseases. Twenty-four compounds were selected based on their algorithm 1 and 2 values being near or above the average of the positive control. After retesting, 9 final hit compounds were selected (Supplemental Figure 2, A and B). These 9 compounds were also distinct from negative controls and aligned with the gentamicin-positive control group (Figure 1D).

To our knowledge, 2 of the 9 hit compounds (methazolamide and clomiphene) have previously been reported to ameliorate the disease phenotype of the $m d x$ mouse model. Methazolamide was identified in a drug screening of the $C$. elegans model, and it was shown to increase the tetanic force in $m d x$ mice (12). Clomiphene is an analog of tamoxifen that was shown to increase force production and suppress fibrosis in $m d x$ mice $(12,24)$. The identification of methazolamide and clomiphene by our 2 algorithms supported the validity of our screening efforts and data analysis.

Secondary and tertiary screening to obtain 2 final hit compounds. To further evaluate the efficacy of the 9 hit compounds and determine their optimal concentrations, we performed an 8-point dose-response assay based on 3 parameters: anti-MyHC immunocytochemistry, anti- $\alpha$-actinin immunocytochemistry, and average cell length (Supplemental Figure 3, A and B). We determined the final hit compounds by their ability to generate dose-dependent response curves with at least 2 of the 3 parameters. Based on the 
A D2 myoblasts

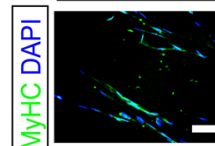

Saponin Q.

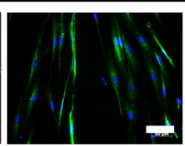

Fenofibrate

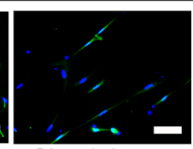

Clomiphene

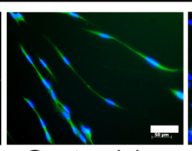

Gentamicin

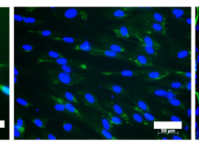

DMSO neg control

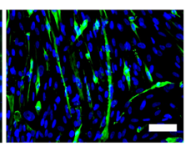

Healthy hiPSC derived myoblasts
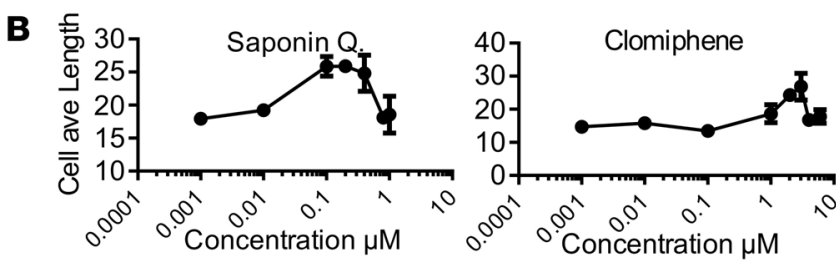

C
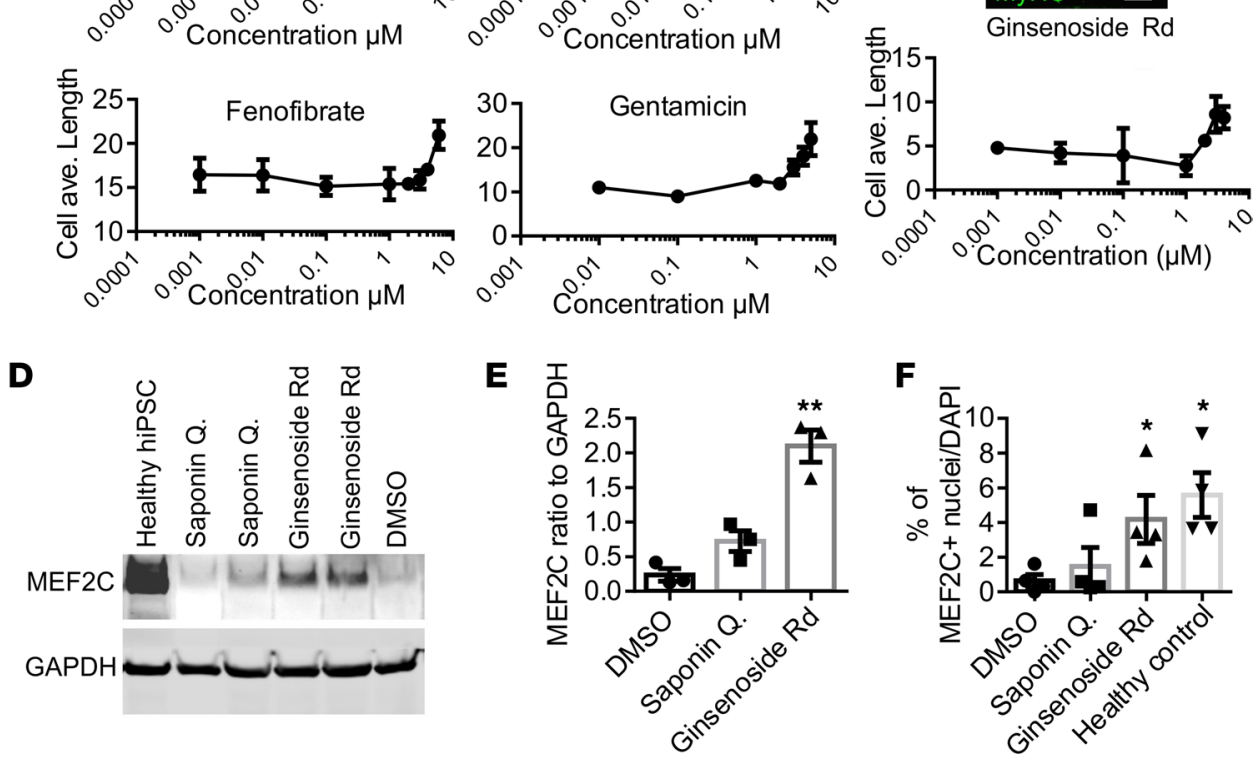

Figure 2. Secondary and tertiary screening to narrow list of candidate compounds to final $\mathbf{2}$ hits. (A) Representative images of saponin Q-, fenofibrate-, clomiphene-, gentamicin-, and DMSO-treated D2 myoblasts, along with healthy hiPSC-derived myoblasts showing MyHC-positive myotubes. (B) Cell average length dose-response curves for saponin Q, fenofibrate, clomiphene, and gentamicin, $n=3$. (C) Representative image of ginsenoside Rd-treated myoblasts labeled with $\mathrm{MyHC}$, and cell average length dose-response curve of ginsenoside Rd; $n=3$. Scale bars: $50 \mu \mathrm{m}$. (D and E) Western blot quantification of MEF2C protein level after saponin $Q$ and ginsenoside $R d$ treatment alongside healthy hiPSC-derived myoblast controls and DMSO negative controls, $n=3$ for all 3 groups; ${ }^{* *} P \leq 0.01$. (F) Quantification of MEF2C-expressing nuclei of saponin Q- and ginsenoside Rd-treated D2 myoblasts; $n=3$ for all 4 groups; ${ }^{*} \leq 0.05$. Data are presented as mean \pm SEM; $\mathbf{E}$ and $\mathbf{F}$ : each point represents an experimental repeat, 1-way ANOVA with Dunnett's multiple-comparisons test with DMSO negative control.

dose-response curves, we selected 3 hit compounds: clomiphene, saponin $Q$ (saponin from quillaja bark), and fenofibrate (Figure 2, A and B, and Supplemental Figure 3, A and B). As the analog of clomiphene, tamoxifen, is already in clinical trials for DMD treatment, we did not further pursue clomiphene (24). Saponin $\mathrm{Q}$ and fenofibrate were selected for further analysis. However, saponin $\mathrm{Q}$ has relatively high toxicity among all members of the saponin family (25), and thus we observed cell death after high-concentration treatment. Therefore, we tested 4 analogs of saponin (akebia, soya saponin, sarsasapogenin, and ginsenoside Rd) (Figure 2C and Supplemental Figure 3, C-E). Among these, ginsenoside Rd treatment not only had a dose-dependent effect on D2 myoblasts, but also increased the levels of MEF2C protein expression (26), whereas saponin Q did not (Figure 2, D-F, and Supplemental Figure 4A). Therefore, ginsenoside $\mathrm{Rd}$ holds advantages over saponin $\mathrm{Q}$ not only due to its lower toxicity, but also because it improved MEF2C expression, which is critical for myogenic differentiation. When fenofibrate and ginsenoside Rd were tested in other DMD hiPSC-derived myoblasts carrying different mutations (GM05127 05533G $>$ C and GM05114 Ex45del), both hit compounds improved myotube formation, as indicated by increased average cell length and $\mathrm{MyHC}$ antibody signal intensity (Figure 3, A and B). As a result, our selected final lead compounds were fenofibrate and ginsenoside Rd (Supplemental Figure 4B). 
A

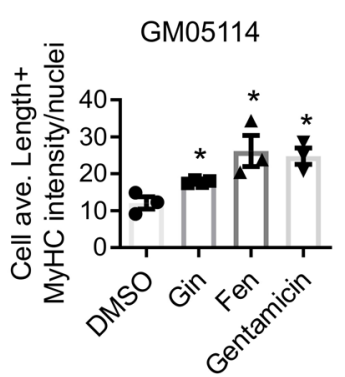

B

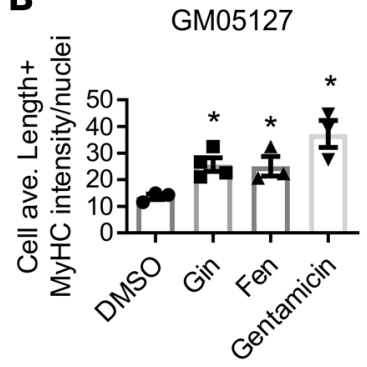

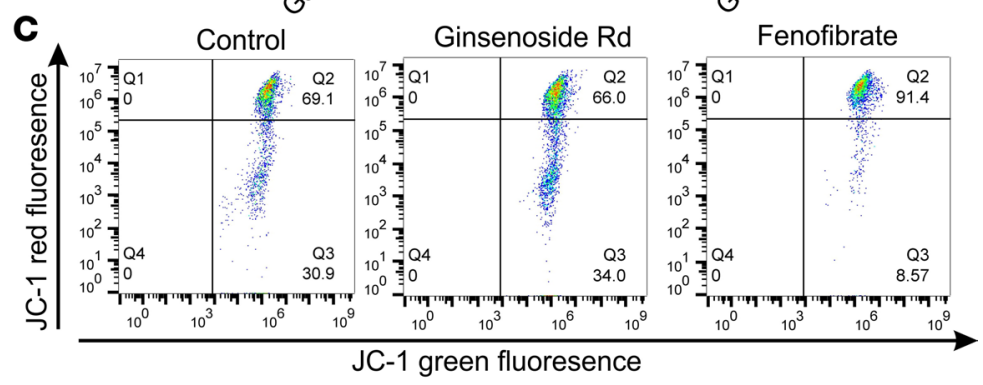

D

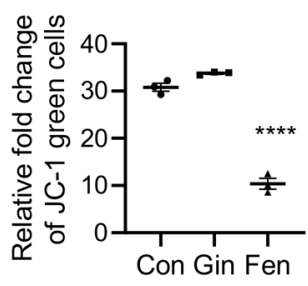

$\mathbf{F}$

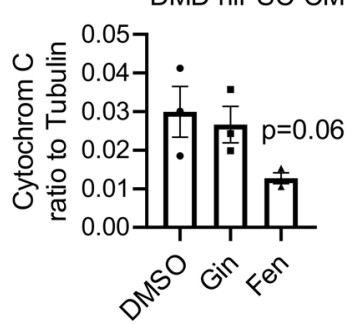

$\mathbf{E}$

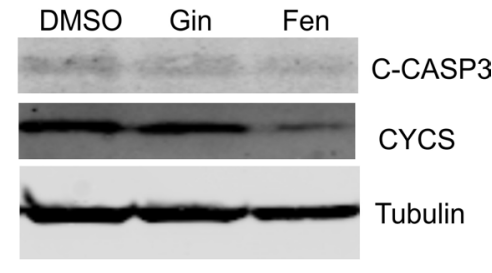

G

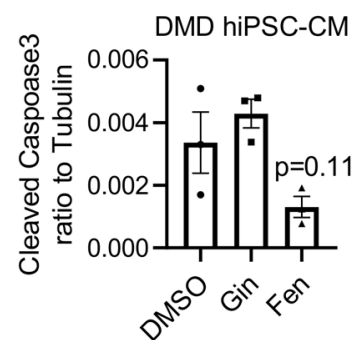

Figure 3. Validation of the effect of fenofibrate and ginsenoside Rd addition on DMD hiPSC-derived myoblasts and DMD-CMs. (A and B) Combination of parameters of cell average length and MyHC antibody immunofluorescence intensity measured from DMD hiPSC-derived myoblasts with mutations other than D2 - GM05114 (Ex45del) and GM05127 (c.5533G>T) - after ginsenoside Rd (Gin; $5 \mu \mathrm{M}$ ), fenofibrate (Fen; $8 \mu \mathrm{M}$ ), and gentamicin treatment; $n=3$ for all 4 groups; ${ }^{*} P \leq 0.05$. (C) Representative flow cytometry plot of JC-1 dye-stained DMD hiPSC-CMs treated with DMSO (control), ginsenoside $\mathrm{Rd}(5 \mu \mathrm{M})$ and fenofibrate $(5 \mu \mathrm{M})$ performed in triplicate. (D) Change in red fluorescence in green JC-1-stained mitochondria, indicating the membrane potential change. Data were generated from DMD-CMs treated with DMSO (control), ginsenoside Rd $(5 \mu \mathrm{M})$, and fenofibrate $(5 \mu \mathrm{M})$ for 7 days, $n=3$ for all 3 groups; ${ }^{* * *} P \leq 0.0001$. (E-G) Western blot quantification of cytochrome $c$ and cleaved caspase-3 in DMD-CMs treated with ginsenoside Rd (5 $\mu \mathrm{M})$, fenofibrate $(8 \mu \mathrm{M})$, or DMSO; $n=3$ for all 3 groups. Data are presented as mean \pm SEM; each point represents an experimental repeat; 1-way ANOVA with Dunnett's multiple-comparisons test with DMSO control.

Fenofibrate treatment is beneficial to DMD hiPSC-derived CMs. Next, we sought to determine whether the 2 hit compounds had any effects on cardiac cells. It was previously reported that CMs derived from DMD iPSCs are associated with increased mitochondrion-mediated cell apoptosis (27). Thus, we evaluated mitochondrial membrane potential in DMD hiPSC-derived CMs (DMD-CMs) treated with the hit compounds using MitoProbe JC-1 dye. JC-1 dye exhibits potential-dependent accumulation in mitochondria, indicated by a fluorescence emission shift from green to red. Consequently, mitochondrial depolarization is indicated by a decrease in the red/green fluorescence intensity ratio. Treatment of CMs with fenofibrate $(5 \mu \mathrm{M})$ for 7 days significantly increased the red/green ratio compared with untreated CMs, indicating 
Table 1. Top pathways selected by IPA of microarray result from D2 myoblasts treated with ginsenoside Rd and fenofibrate for $\mathbf{2 4}$ hours

\begin{tabular}{lc}
\multicolumn{1}{c}{ Ingenuity canonical pathway } & Z score \\
\hline Ginsenoside Rd treatment & $\mathbf{2 . 2 3 6}$ \\
FLT3 signaling & $\mathbf{2}$ \\
Role of CHK proteins in cell cycle checkpoint control & $\mathbf{2}$ \\
Role of IL-17F in allergic inflammatory airway diseases & 1.604 \\
cAMP-mediated signaling & 1.606 \\
PKA signaling & 1.508 \\
ERK/MAPK signaling & \\
\hline TGF- $\beta$ signaling & $\mathbf{2 . 4 4 9}$ \\
NO signaling in the cardiovascular system & $\mathbf{2 . 1 2 1}$ \\
AMPK signaling & 1.414 \\
cAMP-mediated signaling & 1.342 \\
Absolute $Z$ score values of at least 2 are highlighted in bold. &
\end{tabular}

that fenofibrate reduces mitochondrial depolarization and apoptosis (Figure 3, C and D, and Supplemental Figure 5A). However, ginsenoside Rd (5 $\mu \mathrm{M})$ treatment did not have any effect on DMD-CM mitochondrial membrane potential (Figure 3C). In healthy hiPSC-derived CMs we observed an effect similar to that in DMD-CMs when treated with fenofibrate (data not shown). Mitochondria dysfunction is also related to cytochrome $c$ release and caspase- 3 cleavage, which indicate mitochondrial damage and apoptosis (28). In concert with the reduced mitochondrial membrane potential, fenofibrate-treated DMD$\mathrm{CM}$ had a trend toward reduced cytochrome $c$ and slight reduction in cleaved caspase-3 (Figure 3, E-G). On the other hand, healthy hiPSC-derived CMs showed no change or trend toward reduced cytochrome $c$ or cleaved caspase-3 (Supplemental Figure 5, B-D). In summary, fenofibrate but not ginsenoside Rd improved the mitochondrial membrane potential and inhibited apoptosis in DMD-CMs.

Fenofibrate and ginsenoside function through TGF- $\beta$ and ERK1/2 signaling pathways in DMD patient hiP$S C$-derived myoblasts. In order to elucidate the mechanism by which the 2 final hit compounds ameliorated fusion defects of the D2 myoblasts, we performed unbiased global transcriptional profiling on D2 myoblasts treated with fenofibrate $(8 \mu \mathrm{M})$ or ginsenoside $\mathrm{Rd}(5 \mu \mathrm{M})$. A heatmap showed distinctive gene expression profiles among groups (Supplemental Figure 6A), and the transcriptional analysis results were validated by quantitative (qPCR) (29) (Supplemental Figure 6, B and C). Ingenuity pathway analysis (IPA) showed that the most significantly positive correlation $(Z$ score $>2)$ after ginsenoside $R d$ treatment was with the FLT3 signaling pathway (Table 1). It has been reported that FLT3 regulates myogenic differentiation by enhancing the expression of p21 (WAF1/CIP1), a cell cycle inhibitor, resulting in cells exiting the cell cycle (30). We detected increased levels of p21 in D2 myoblasts under FLT3 treatment, and this effect was also seen in ginsenoside Rd-treated D2 myoblasts (Figure 4, A and B). At the same time, ERK1/2, a known FLT3 pathway downstream effector (31), was activated both by FLT3 recombinant protein and ginsenoside Rd in D2 myoblasts (Figure 4, C and D) (32). Fenofibrate treatment suppressed TGF- $\beta$ signaling in D2 myoblasts $(Z$ score $<-2)$, as determined by IPA (Table 1$)$. Moreover, when D2 myoblasts were treated with a combination of fenofibrate and TGF- $\beta 1$ recombinant protein, SMAD2/3 phosphorylation was reduced, indicating that fenofibrate suppressed TGF- $\beta$ signaling (Figure 4, E and F) (33). As TGF- $\beta$ signaling plays a suppressive role in muscle differentiation, it is likely that fenofibrate improves D2 myoblast differentiation/fusion efficiency by inhibiting TGF- $\beta$ (34). At the same time, we observed an inhibitory effect of fenofibrate on primary human fibroblast growth, which could have been caused by suppressed TGF- $\beta$ signaling (Supplemental Figure 6D). Based on the results above, we conclude that the positive effects of ginsenoside Rd and fenofibrate are associated with the FLT3 and TGF- $\beta$ pathways, respectively.

Ginsenoside $R d$ and fenofibrate ameliorate the disease phenotype of the mdx mouse model of DMD. Since ginsenoside Rd and fenofibrate were effective in correcting the in vitro DMD phenotype of DMD hiPSCderived myoblasts, we sought to determine whether they also had a therapeutic effect in vivo. We tested 
A

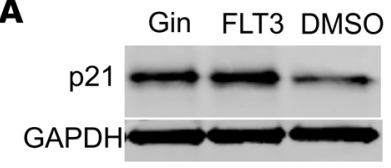

B

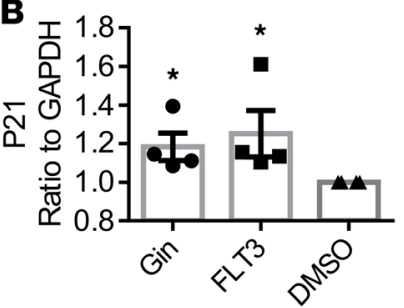

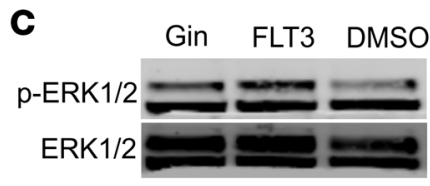

D

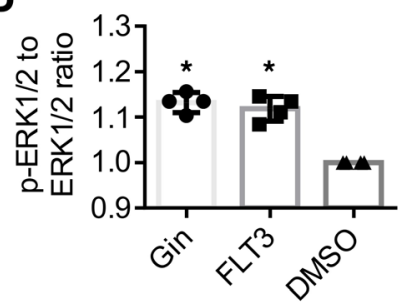

$\mathbf{F}$

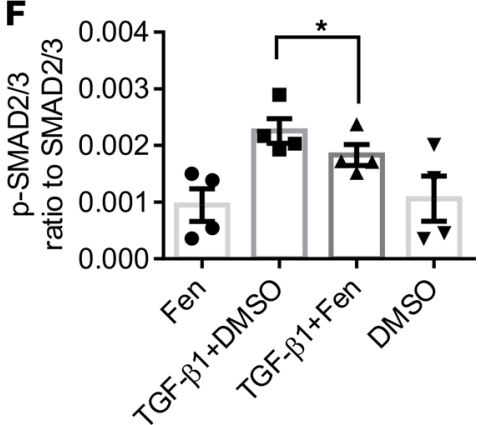

Figure 4. Two selected compounds, ginsenoside Rd and fenofibrate, function via FLT3 signaling and TGF- $\beta$ signaling, respectively. (A and B) Quantification of Western blot of p21 protein expression in D2 myoblasts treated with ginsenoside Rd $(25 \mu \mathrm{M})$, FLT3 recombinant protein $(100 \mathrm{ng} / \mathrm{mL})$, or DMSO for 30 minutes; $n=4$ for all 3 groups; ${ }^{*} P \leq$ 0.05. (C and D) Quantification of Western blot of p-ERK $1 / 2$ in D2 myoblasts treated with ginsenoside (5 $\mu M$ ), FLT3 recombinant protein ( $100 \mathrm{ng} / \mathrm{mL}$ ), or DMSO for 30 minutes; $n=4$ for all 3 groups; ${ }^{*} P \leq 0.05$. (E and $\mathbf{F}$ ) Quantification of Western blot of p-SMAD2/3 after treatment with fenofibrate $(8 \mu \mathrm{M})$ and/or TGF- $\beta 1$ recombinant protein (40 ng/ $\mathrm{mL}$ ) for 24 hours; $n=4$ for all 4 groups, ${ }^{*} P \leq 0.05$. Data are presented as mean $\pm S E M$; each point represents an experimental repeat. B and D: 1-way ANOVA with Dunnett's multiple-comparison tests with DMSO negative control; F: 1-way ANOVA with Tukey's multiple-comparisons test).

each of the 2 compounds in $m d x^{5 v}$ mice (35). We treated the mice with ginsenoside $\mathrm{Rd}(10 \mathrm{mg} / \mathrm{kg})$ through daily intraperitoneal injections and fenofibrate $(0.1 \% \mathrm{wt} / \mathrm{wt})$ through diet beginning postnatal day 21 , for 8 weeks (36) (Figure 5A). Neither of the compounds affected the growth curve of the $m d x$ mice (Figure 5B). At the end of the treatment period, the pathology of different muscles was assessed. Skeletal muscle fibrosis is most prominent in the diaphragm of $m d x$ mice (37), and the levels of fibrosis in diaphragm were reduced by treatment with ginsenoside Rd (29\%) and fenofibrate (42.1\%) (Figure 5, C and D, and Supplemental Figure 7A). Decreased levels of fibrosis were further confirmed by quantifying hydroxyproline, which is a modified amino acid found in fibrosis. Fenofibrate treatment reduced hydroxyproline in the diaphragm by 29\% (Supplemental Figure 7B). Numbers of Evans blue dye-positive (EBD-positive) fibers (a marker of cell membrane damage) and centrally nucleated fibers (a marker of muscle degeneration and regeneration) were not significantly different in treated muscles (Supplemental Figure 7, C and D). Cross-sectional area (CSA) analysis of TA muscle showed that the number of very small fibers $\left(0-1000 \mu \mathrm{m}^{2}\right)$ decreased and the medium-sized fibers (1000-2000 $\left.\mu \mathrm{m}^{2}\right)$ increased in both ginsenoside $\mathrm{Rd}-$ and fenofibrate-treated $m d x$ when with sham-treated control mice (Figure 5E). Mean CSA was not significantly different across treatment groups (Supplemental Figure 7E). However, the standard deviations of the ginsenoside Rd-treated group, 1476, and fenofibrate-treated group, 1315, were lower than that of the sham-treated group, 1588, indicating that both compounds reduced the variability of the fiber sizes.

We performed a series of functional assessments, including grip strength and treadmill running endurance. Both fenofibrate and ginsenoside Rd significantly improved $m d x$ mouse forelimb grip strength, by $16 \%$ and 19\%, respectively (Figure $5 \mathrm{~F}$ ). Fenofibrate treatment improved endurance in treadmill running by $50 \%$ compared with control treatment in $m d x$ mice, while ginsenoside Rd had no effect on treadmill 
A
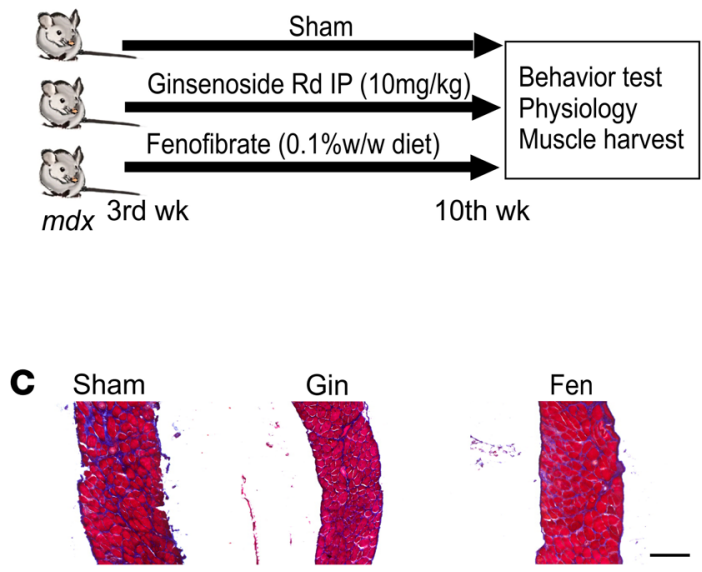

$\mathbf{E}$
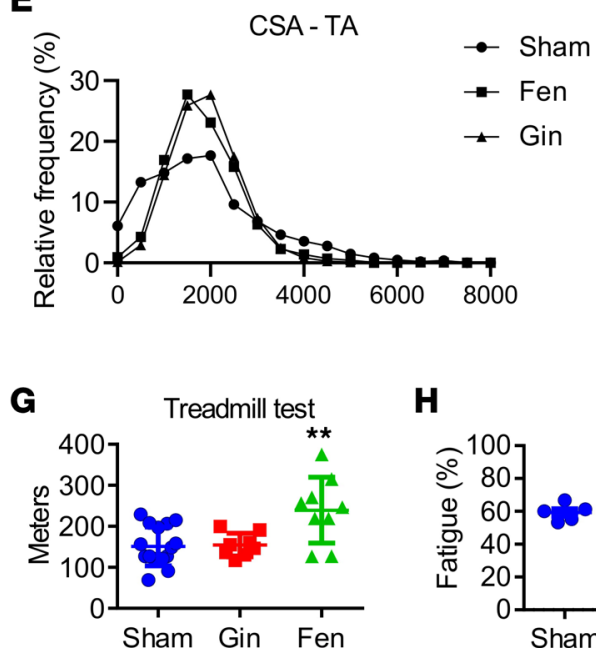

H

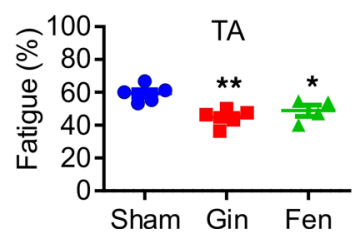

B

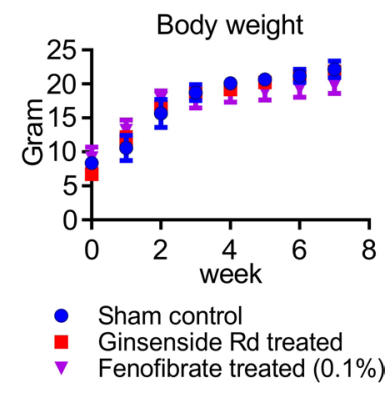

D

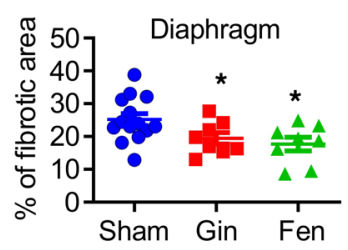

$\mathbf{F}$

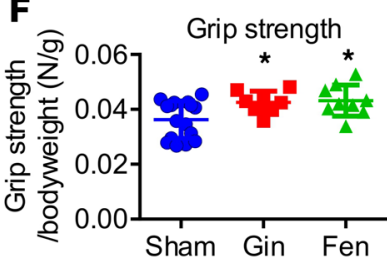

I

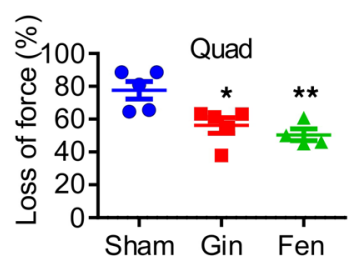

Figure 5. Ginsenoside Rd and fenofibrate treatment ameliorate the disease phenotype of $\boldsymbol{m d} \mathbf{x}^{5 \mathrm{cv}}$ mice. (A) Scheme showing compound treatment of $m d x$ mice. (B) Body weight of $m d x$ mice measured weekly from 3 to 10 weeks of age. Sham control was $m d x$ mice treated with standard diet; $n=7$ for all 3 groups. (C and D) Staining and quantification of Masson's trichrome-labeled fibrotic area (blue) in diaphragm muscle of mice; $n$ (sham) $=14, n$ (ginsenoside Rd) $=8, n$ (fenofibrate) $=8$; ${ }^{*} \leq 0.05$; scale bar: $200 \mu \mathrm{m}$. (E) Frequency distribution of muscle fiber CSA of TAs; $n=5$ for all 3 groups. (F) Measurement of forelimb grip strength normalized to body weight of $m d x$ mice treated with ginsenoside Rd or fenofibrate; $n$ (sham) = 16, $n$ (ginsenoside Rd) = 8, $n$ (fenofibrate) $=9 ;{ }^{*} P \leq 0.05$. (G) Maximum distance $m d x$ mice ran on treadmill; $n\left(\right.$ sham) $=16, n$ (ginsenoside Rd) $=8 ; n$ (fenofibrate) $=9 ;{ }^{* *} P \leq 0.01$. (H) Fatigue index (\%) represents the reduction in maximal tetanic tension measured after 5 minutes of repeated tetanic stimulation at $1 \mathrm{~Hz}$ in TA muscle of $m d x$ mice; $n=5$ for all 3 groups; ${ }^{*} P \leq 0.05$, ${ }^{*} P \leq 0.01$. (I) Susceptibility to injury (percent loss of maximal isometric force after lengthening contractions) of quadriceps muscle (Quad) $n=5$ for all 3 groups; ${ }^{*} P \leq 0.05,{ }^{* *} P \leq 0.01$. Data are presented as mean $\pm \mathrm{SEM}$; each point represents 1 mouse; 1-way ANOVA with Dunnett's multiple-comparisons test with sham control.

running (Figure 5G and Supplemental Figure 7F). In in situ physiology testing, there was no significant difference in maximal isometric force generated by tibialis anterior (TA) or quadriceps muscle with either treatment compared with the sham control (Supplemental Figure 7, G and H). However, repeated maximal isometric contractions showed that TA muscles of mice treated with fenofibrate or ginsenoside Rd were less susceptible to fatigue compared with untreated $m d x$ TA muscles (Figure $5 \mathrm{H}$ ). As in previous studies, $m d x$ quadriceps muscles were highly susceptible to injury ( $78 \% \pm 5.3 \%$ loss in muscle force after injury), but treatment with ginsenoside $\mathrm{Rd}$ or fenofibrate resulted in significantly less contraction-induced injury $(56 \%$ $\pm 4.8 \%$ and $50 \% \pm 3.5 \%$ loss in muscle force, respectively) (Figure $5 \mathrm{I}$ ).

Overall, our results indicate that ginsenoside $\mathrm{Rd}$ and fenofibrate treatment ameliorated disease phenotypes in $m d x$ mice. 


\section{Discussion}

Drug discovery and repurposing for treatment of DMD has mostly employed 2 strategies: restoring dystrophin expression; and modifying downstream pathological pathways, including inflammation, fibrosis, and oxidative stress (38). A rapid and relevant method to identify disease-modifying treatments for DMD could enable a swift translational process, from drug screening to therapy. To improve drug discovery and repurposing of known or approved drugs for DMD, human dystrophic muscle cells that demonstrate a distinguishable phenotype are needed. Recently, the use of hiPSCs has gained interest as an emerging approach in drug discovery for genetic diseases (39). hiPSCs provide a scalable source of starting material that can be easily used in drug screens for DMD. Despite this advantage, to our knowledge there have been no reported applications of hiPSCs for DMD drug screens due to the lack of efficient and reproducible DMD hiPSC models.

During the past 4 years, protocols have emerged for myogenic differentiation of DMD hiPSCs. In 2016, 2 groups, including ours, independently reported protocols to directly differentiate hiPSC using a chemically defined system $(15,16)$. Applying Wnt activator and Notch inhibitor, Choi et al. depicted a distinct transcription profile and phenotype of DMD hiPSC-derived myoblasts from the healthy hiPSCderived myoblasts. With Wnt activation and BMP inhibition, Chal et al. reported that myotubes formed from myoblasts derived from $m d x$ mice presented abnormal branching. While both differentiation protocols showed myogenic commitment and ex vivo contraction of skeletal muscle myotubes, Hicks et al., using Chal's protocol, did not report fusion defects in myoblasts derived from DMD hiPSCs following $\mathrm{NCAM}^{+} /$ $\mathrm{HNK}^{-}$purification (40). The discrepancy in reported phenotypes of $\mathrm{NCAM}^{+} / \mathrm{HNK}^{-} \mathrm{DMD}$ hiPSCderived myoblasts could be due to the fact that the IGF-1 and HGF growth factors, which were used in Chal's protocol, can enhance myoblast fusion potential $(41,42)$. In comparison, our myoblasts culture system does not contain any growth factors, demonstrating the native myotube formation potential. Moreover, our transcriptional and translational profile data showed increased BMP and TGF- $\beta$ signaling in DMD hiPSC-derived myoblasts. A similar phenomenon was found in myoblasts isolated from DMD patient biopsies, and these myoblasts also demonstrated limited growth capability (17). Using the hiPSC differentiation method described above, we developed an imaging-based screening system in which myotube formation was visualized by staining chemically induced DMD iPSC-derived myoblasts with antibodies. This straightforward, easily detectable phenotype via imaging can be used in future compound library screens.

The JHCCL used in this study contains around 1000 FDA-approved and 500 foreign agency-approved compounds. While designing and testing a new drug is costly and time consuming, screening approved drugs for previously unidentified activities could significantly speed the process of drug development. Using this compound library together with the imaging-based screen system described above, we selected 2 final compounds - ginsenoside Rd and fenofibrate. Ginsenosides are a group of active components found in Panax ginseng, a well-known herbal medicine touted to improve thinking, concentration, memory, work efficiency, physical stamina, and athletic endurance (43). Although the therapeutic potential of ginseng has been studied extensively, ginsenosides, which belong to the saponin family, have not yet been thoroughly investigated. Ginsenosides are reported to function primarily via antiinflammatory and antioxidant effects (44). Ginsenoside Rd was chosen among all the ginsenosides because of its function of inhibiting calcium influx (a hallmark of DMD pathology), inhibiting ROS, decreasing cellular apoptosis, and stabilizing mitochondrial membrane potential (45). In this study, we found that ginsenoside Rd helped restore fusion of DMD hiPSC-derived myoblasts. We performed gene ontology analyses using microarray results from DMD hiPSC-derived myoblasts treated with ginsenoside Rd to uncover this drug's mechanism of action. When analyzing the pathways affected by ginsenoside Rd treatment, the FLT3 pathway was the top most significantly regulated pathway. FLT3 is a type III tyrosine kinase, and its mutation in leukemia results in aberrant cell growth (46). To date, there has been only one study to our knowledge reporting FLT3 as necessary for myogenic differentiation; overexpression of FLT3 appeared to promote cell cycle exit and myoblast fusion by inhibiting ERK activity through p120RasGAP (30). In our study, enhanced ERK phosphorylation was observed in D2 myoblasts by recombinant FLT3 $(100 \mathrm{ng} / \mathrm{mL})$ treatment as well as ginsenoside $\mathrm{Rd}(20 \mu \mathrm{M})$ treatment (Figure 4, C and D), which is in contrast to the result of Ge et al. (30). There are a couple of potential explanations. In leukemia, activated FLT3 receptor is known to induce RAS/ERK activation (47). Additionally, several reports have claimed that ERK phosphorylation is induced when myoblasts are terminally differentiated into myotubes $(48,49)$. Therefore, FLT3 is more likely to activate ERK and induce D2 myoblast differentiation upon recombinant FLT3 or ginsenoside Rd treatment. In this study, when ginsenoside Rd was given to $m d x$ mice, we observed that ERK was activated, as suggested by 
an enhanced p-ERK1/2 protein level (Supplemental Figure 8, C and D). At the same time, ginsenoside $\mathrm{Rd}$ treatment improved forelimb grip strength and increased resistance to fatigue. This outcome could be the result of ginsenoside Rd's combined effects, including its antiinflammatory and antioxidant activity in muscle as well as promotion of myotube differentiation.

The other identified compound, fenofibrate, is a well-established drug for treatment of hypertriglyceridemia, low HDL-C levels, or dyslipidemia. Fenofibrate's metabolite - fibrate acid — is a PPAR $\alpha$ agonist that can regulate fatty acid metabolism genes to reduce LDL, total cholesterol, and triglycerides, and to increase high-density lipoprotein (HDL) (50). In our study, when $m d x$ mice were administrated $0.1 \%$ wt/wt fenofibrate diet, they showed decreased triglyceride levels (Supplemental Figure 8A) and increased HDL levels (Supplemental Figure 8B), similar to what has been reported in humans (51). There have been reports suggesting that fenofibrate is beneficial to muscle function. In one study, fenofibrate was shown to decrease glucocorticoid levels, thereby preventing muscle wasting in small cell lung cancer patients (52). Another study showed that fenofibrate administration in arthritic rats inhibited expression of myostatin in skeletal muscle, prohibiting muscle atrophy (53). Our examination demonstrated that fenofibrate inhibited TGF- $\beta$ signaling activity (Figure $4, \mathrm{E}$ and F). TGF- $\beta$ signaling has been extensively studied in the context of muscular dystrophy due to its role as a negative regulator of muscle growth and inducer of fibrosis (54). Although we did not detect inhibited TGF- $\beta$ signaling in quadriceps muscle in $m d x$ mice after fenofibrate treatment (Supplemental Figure 8, E and F), we observed decreased fibrosis and increased muscle function in fenofibrate-treated $m d x$ mice. Moreover, proliferation of fibroblasts was inhibited by fenofibrate treatment in vitro (Supplemental Figure 5D) (55), supporting the hypothesis that the mechanism underlying fenofibrate's effects is inhibition of TGF- $\beta$ signaling.

Apart from skeletal muscle, the heart is also significantly affected in DMD and is a major cause of mortality. CMs that lack dystrophin protein are vulnerable to membrane fragility, resulting in cell death, which is followed by fibrosis of the heart (56). Therefore, we performed compound treatment of DMD-CMs, which were reported to recapitulate the DMD disease phenotype by exhibiting increased cytosolic $\mathrm{Ca}^{2+}$ and mitochondria damage. We found that fenofibrate reduced mitochondrial depolarization, indicating that it may have therapeutic benefits for cardiac muscle in addition to skeletal muscle (27).

In summary, our study shows the application of hiPSC-derived myoblasts in a high-content imagingbased drug screening platform to discover 2 compounds, ginsenoside Rd and fenofibrate. These 2 compounds ameliorated the dystrophic phenotype in the $m d x$ mouse model. In treadmill and grip strength assays, fenofibrate-treated $m d x$ mice ran longer distances, and mice treated with either compound had enhanced forelimb grip strength (Figure 5, F and G). In physiology testing, TA muscle of $m d x$ mice treated with either compound showed significantly less susceptibility to muscle fatigue (Figure $5 \mathrm{H}$ ), a major symptom of DMD. Although specific force of TA and torque of quadriceps did not improve, $m d x$ mice treated with either fenofibrate or ginsenoside $\mathrm{Rd}$ showed a significant reduction in susceptibility to contraction-induced injury (Figure 5I). The above evidence raises the possibility that these drugs could be tested in the context of DMD. The results of this study indicate the feasibility of using hiPSC-based medium-scale drug screening to identify FDA-approved drugs or natural products for orphan diseases.

\section{Methods}

Animal and treatment. $m d x^{5 c v}$ (B6Ros.Cg-Dmdmdx-5Cv/J) mice were obtained from the Jackson Laboratory. Mice were maintained in a 12-hour/12-hour dark light cycle (7 am-7 pm) with ad libitum access to food and water. Male $m d x$ mice at 3 weeks of age were randomly assigned to one of the 3 groups: sham, fenofibrate, or ginsenoside Rd. Mice received a standard chow diet (Global 18\% Protein Rodent Diet-Control, Teklad) or a diet containing fenofibrate (0.1\%, wt/wt, MilliporeSigma) (57) mixed into the standard chow. Ginsenoside $\mathrm{Rd}$ was suspended in saline containing 10\% 1,3-propanediol as vector. Ginsenoside $\mathrm{Rd}$ (10 $\mathrm{mg} / \mathrm{kg}$ ) (58) was provided to $m d x$ mice through intraperitoneal injection. The sham group received standard chow diet and intraperitoneal injection of vector at the same time.

Generation of DMD patient iPSCS. D2325 fibroblasts were obtained from a DMD patient at the Kennedy Krieger Institute. Genetic testing revealed that this patient had a stop codon mutation at c.457. Other fibroblasts were purchased from Coriell Institute for Medical Research (GM05127 and GM05114) with appropriate Material Transfer Agreement documents. Human cells were cultured in DMEM containing 10\% FBS. Fibroblasts were plated onto 24-well plates and reprogrammed with a CytoTune-iPS Sendai Reprogramming Kit (Invitrogen) with the standard protocol. After 9 days, cells were seeded onto a mouse embryonic fibroblast (MEF) feeder layer. 
iPSC differentiation and myoblast maintenance. The DMD hiPSC-derived myoblasts were differentiated using the CHIR-DAPT protocol (15). Briefly, hiPSCs were plated as single cells on Geltrex-treated (Gibco) dishes at a density of $1.5 \times 10^{5}$ cells per well in a 24-well plate, in the presence of MEF-conditioned N2 media containing $10 \mathrm{ng} / \mathrm{mL}$ FGF-2 (PeproTech) and $10 \mu \mathrm{M}$ Y-27632 (Cayman). The cells were induced to differentiate into myoblasts by adding CHIR99021 (3 $\mu \mathrm{M})$ in N2 medium for 4 days and DAPT (10 $\mu \mathrm{M})$ for the following 8 days. Cells continued to differentiate and mature in N2 medium for the next 13 days. Myoblasts were collected by FACS with the selection marker NCAM ${ }^{+} / \mathrm{HNK}^{-}$(NCAM: 5.1H11, Developmental Studies Hybridoma Bank [DSHB]; HNK1: C6680, MilliporeSigma).

The $\mathrm{NCAM}^{+} / \mathrm{HNK}^{-}$myoblasts were maintained in a humidified incubator containing $5 \% \mathrm{CO}_{2}$ at $37^{\circ} \mathrm{C}$ and grown in N2 media supplemented with $10 \%$ FBS. To induce myotube formation, expanded $\mathrm{NCAM}^{+} \mathrm{HNK}^{-}$myoblasts were plated to confluence and switched to N2 media without serum.

Drug screen. The JHCCL (v1.3) was provided by David Sullivan of the Johns Hopkins Bloomberg School of Public Health. The FACS-sorted DMD hiPSC-derived myoblasts were seeded at 25,000 cells per well in 96-well plates. Medium was changed to N2 without additional serum, and cells were treated with compounds from the JHCCL $(1 \mu \mathrm{M})$, DMSO $(0.1 \%$, negative control), or gentamicin $(500 \mu \mathrm{g} / \mathrm{mL}$, MilliporeSigma) every 3 days for 9 days. The compound library consisted of 1524 small molecules. Cells were then fixed with 4\% paraformaldehyde and stained with MyHC antibody (2235587, DSHB) $(59,60)$. Automated image acquisition protocol with high-content imaging/analyzing system (BD Pathway 855, at ChemCore, Johns Hopkins School of Medicine), and automated analysis programming (BD AttoVision) were used to image and analyze myotube formation. Primary hits were validated in secondary replicate experiments $(n=3)$. Antibodies used for immunofluorescence in secondary as well as tertiary screen were MEF2C (1410781, MilliporeSigma) and $\alpha$-actinin (A7811, MilliporeSigma).

Western blot. Whole-cell extracts were prepared by lysing cells on a plate with RIPA buffer (Cell Signaling Technology [CST]) supplemented by proteinase inhibitor and phosphatase inhibitor cocktail (CST). Western blotting was performed according to the standard protocol using precast NuPAGE (4\%-12\%) BisTris gel (Invitrogen). Protein transfer was performed with the Bio-Rad Turbo or wet/tank blotting system. Nitrocellulose membranes were incubated with primary antibodies overnight at $4^{\circ} \mathrm{C}$. Membranes were then incubated with a secondary IRDye 800-conjugated anti-rabbit IgG or Alexa Fluor 680-anti-mouse $\mathrm{IgG}$, and proteins were visualized and quantified using the LI-COR Odyssey Infrared Imaging System. Primary antibodies used in Western blotting were p-ERK1/2 (9101, CST), ERK (9102, CST), p-SMAD2/3 (8828, CST), SMAD2/3 (3102, CST), p21 (2947, CST), and MEF2C (1410781, MilliporeSigma).

Affymetrix microarray and $q P C R$. Triplicate samples were used in microarray analysis. D2 hiPSC-derived myoblasts were treated with ginsenoside $(5 \mu \mathrm{M})$, fenofibrate $(8 \mu \mathrm{M})$, or DMSO for 24 hours in the differentiation medium. RNA was isolated using TRIzol (Invitrogen Life Technologies), followed by purification and DNase digestion using RNeasy Mini Kits (QIAGEN) according to the manufacturer's instructions. Quantification of total RNA was performed on a NanoDrop spectrophotometer (Thermo Fisher Scientific), and RNA quality was tested on an Agilent TapeStation with R6K ScreenTapes (RNA integrity number [RIN] 7.6-9.8). Generation of sense strand cDNA from purified total RNA was followed by second-strand synthesis, in vitro transcription cDNA synthesis, and single-stranded cDNA synthesis and RNA hydrolysis. Fragmentation and labeling were performed according to the manufacturer's instructions (GeneChip WT PLUS Reagent Kit, Affymetrix). RNA extraction and qPCR were performed according to previous protocols (61), and primers are listed in Supplemental Table 1. The microarray data have been deposited in the NCBI's Gene Expression Omnibus database (GEO GSE121023).

Hierarchical clustering was performed using Spotfire Genomics Suite with Functional Genomics v9.1.2 (TIBCO). Clustering of the 1042 mean-subtracted values used the unweighted average (unweighted pair group method with arithmetic mean [UPGMA]) method on correlation similarity with input rank ordering function. The heatmap (Supplemental Figure 6A) displays the hierarchical clustering of 1042 transcripts selected for having $P$ values less than 0.05 for differentiating among the 3 biological classes: control, fenofibrate, and ginsenoside Rd. Raw microarray CEL files were extracted with Partek Genomics Suite 7.0 and the $\log _{2}$ values quantile normalized with the RMA protocol, then mean-subtracted for each transcript before hierarchical clustering.

Treadmill and grip strength. Forelimb grip strength was measured as maximal tensile force using a computerized force transducer (Grip Strength Meter, Bioseb). Five measurements were performed for each animal, and the maximum value was used for the analysis. Treadmill testing was performed using a motor-driven treadmill (Columbus Instruments). Before the test day, acclimatization was performed 
5 times over a period of 2 weeks at $10 \mathrm{~m} / \mathrm{min}$. On the test day, mice ran at $5 \mathrm{~m} / \mathrm{min}$ for 5 minutes (warmup), and the speed was increased $1 \mathrm{~m} / \mathrm{min}$ every minute up to $10 \mathrm{~m} / \mathrm{min}$. Mice were considered exhausted when they sat for more than 10 seconds on a shock pad for the third time.

EBD staining and histology analysis. Gastrocnemius, TA, and diaphragm muscles were embedded in OCT (Sakura), frozen in isopentane, and cross-sectioned ( 2-10 $\mu \mathrm{m}$ thickness). The sections were also stained with DAPI to visualize nuclei. In mice where $\operatorname{EBD}(10 \mathrm{mg} / \mathrm{mL}$, MilliporeSigma) was used to evaluate membrane damage, EBD was injected at $0.05 \mathrm{~mL} / 10 \mathrm{~g}$ intraperitoneally 24 hours before sacrificing the mice. To evaluate EBD staining, gastrocnemius muscle sections were fixed with acetone and imaged with fluorescence microscopy. The area of EBD as a percentage of total area was calculated. To evaluate the number of central nucleated fibers, sections of the TA muscle were stained with hematoxylin and eosin. Centrally nucleated fiber number was counted and analyzed against total fiber number. Diaphragm sections $(10 \mu \mathrm{m})$ were stained with Masson's trichrome to determine collagen content. The stained areas were quantified against the total area.

In vivo muscle physiology. Quadriceps strength (maximal isometric torque) and susceptibility to injury were assessed in vivo as described previously $(62,63)$. Briefly, animals were anesthetized with $3 \%-5 \%$ isoflurane and placed in a supine position. The thigh was stabilized and the ankle was secured onto a lever arm. The knee was aligned with the axis of the stepper motor (model T8904, NMB Technologies) and a torque sensor (QWFK-8M, Sensotec), and the femoral nerve was stimulated via subcutaneous needle electrodes. A custom program based on commercial software (LabView version 8.5, National Instruments) was used to synchronize contractile activation and the onset of forced knee flexion. The position of the leg that results in optimal muscle length has been previously described, and maximum isometric torque was measured in Newton-millimeters ( $\mathrm{Nmm}$ ). Injury was induced by 15 forced lengthenings (knee flexion) superimposed onto maximal quadriceps contractions through a 40- to 100-degree arc of motion (with full knee extension considered 0 degrees) spaced 1 minute apart. Loss in maximal isometric torque was measured 5 minutes after the last lengthening contraction. Since the knee position, lever arm, and moment arm of the muscle are unchanged between tests, maximal isometric torque reflects maximal isometric muscle force.

TA muscle strength (maximal isometric force) and rate of fatigue were measured as described previously (64). Briefly, animals were anesthetized with $3 \%-5 \%$ isoflurane and placed in a supine position. The tibia was stabilized, and the distal tendon of the TA was surgically released and attached to the load cell (FT03, Grass Instruments). The load cell was adjusted via a micromanipulator to stretch the muscle to resting length (optimal length). TA contraction was then triggered via subcutaneous stimulation of the fibular nerve, and the resulting force generated was sampled at $1 \mathrm{kHz}$ and analyzed with acquisition software (PolyVIEW 16, Grass Instruments). After contractile function experiments, animals were euthanized, and the TAs were harvested and weighed. As muscle length was fixed in all experiments, and muscle density is assumed to be a constant, physiological CSA of the TA was solely a function of muscle mass. Force was therefore normalized to TA mass to calculate as specific force $(g / g)$. To provide an index of fatigue, muscle tension was measured after 5 minutes of tetanic stimulation (200-ms train duration) repeated at 1 $\mathrm{Hz}$ and expressed as a percentage of initial tension.

hiPSC-CM differentiation and JC-1 assay. DMD hiPSC-derived cardiomyocytes (GM05114 Ex45del) were plated as single cells on Geltrex-treated dishes, at a density of $0.5 \times 10^{5}$ to $2 \times 10^{5}$ cells per well in a 6-well plate. Cells were cultured in E8 medium (Gibco) for 2 days before induction to CMs. When cells became $70 \%-90 \%$ confluent, cardiomyocyte differentiation was carried out in RPMI (Gibco) media supplemented with B27 without insulin. Differentiation was induced by addition of $6 \mu$ M CHIR99021 (Selleck Chemicals) to a confluent monolayer of cells for 2 days, followed by addition of $10 \mu \mathrm{M}$ IWR-1 (Enzo Life Sciences) from day 3 to 5. CMs were metabolically enriched from day 8 to 10 in glucose-free DMEM supplemented with lactate $(4 \mathrm{mM})$ as previously described (65). CMs (14 days old) were treated with ginsenoside $\mathrm{Rd}(5 \mu \mathrm{M})$ and fenofibrate $(5 \mu \mathrm{M})$ for 7 days in RPMI media supplemented with B27 and $50 \mu \mathrm{M}$ isoproterenol. Media was changed every 48 hours. JC-1 dye (Mitochondrial Membrane Potential Probe) assay was performed according to the manufacturer's protocol (Invitrogen).

Statistics. All data are shown as mean \pm SEM and were subjected to statistical analysis. Significance was analyzed by 1-way ANOVA using Dunnett's or Tukey's multiple-comparisons test or were analyzed by 2-tailed unpaired Student's t test. $P \leq 0.05$ was considered significant. The $n$ values indicate the number of independent biological samples. Data were analyzed and represented with GraphPad Prism. Investigators were blinded to allocation during animal experiments and outcome assessment. 
Study approval. All human sample-related experiments were approved by the Institutional Stem Cell Research Oversight Committee (ISCRO: RN00000264 For ISCRO00000017) and the Johns Hopkins Medicine Institutional Review Boards (JHM IRBs, NA_00019985), Johns Hopkins University School of Medicine. All animal experiments were approved by the IACUC of the Johns Hopkins University School of Medicine. Written informed consent was received from the patient who provided the D2 sample at the Kennedy Krieger Institute (IRB NA_00019985).

\section{Author contributions}

CS designed and conducted the experiments, analyzed the data, and wrote the manuscript. IYC conducted the cell differentiation. YIRG conducted the experiments. CCT analyzed the microarray data. SRI and RML conducted the $m d x$ physiology experiments and data analysis. PA conducted the iPSC-CM experiments. GL and KRW designed the study. All authors reviewed the manuscript.

\section{Acknowledgments}

We thank members of the Wagner laboratory and the Lee laboratory for valuable discussions. Especially, we thank Hotae Lim (Institute for Cell Engineering, Johns Hopkins University School of Medicine) and Carlo Serra (Center for Genetic Muscle Disorders, Hugo W. Moser Research Institute at Kennedy Krieger Institute) for providing technical support. This work was supported by NIH grants R01NS093213 and R01AR070751, the Maryland Stem Cell Research Fund (MSCRF), the Muscular Dystrophy Association (MDA) and the Global Research Development Center program from the Korea National Research Foundation (all to GL). This work was also funded by Stand Strong, Ryan's Quest, Michael's Cause, and Pietro's Fight (all to KRW); a Maryland Stem Cell Fellowship (to CS); and American Heart Association Career Development Award 19CDA34760161 (to PA).

Address correspondence to: Gabsang Lee, Institute for Cell Engineering, Johns Hopkins University, School of Medicine, Broadway Research Building, 733 North Broadway, Suite 747, Baltimore, Maryland 21205, USA. Phone: 443.287.4436; Email: glee48@jhmi.edu. Or to: Kathryn R. Wagner, Center for Genetic Muscle Disorders, Kennedy Krieger Institute, 707 North Broadway, Baltimore, Maryland 21205, USA. Phone: 443.923.9525; Email: wagnerk@kennedykrieger.org.

1. Ryder S, et al. The burden, epidemiology, costs and treatment for Duchenne muscular dystrophy: an evidence review. Orphanet $J$ Rare Dis. 2017;12(1):79.

2. Angelini C. The role of corticosteroids in muscular dystrophy: a critical appraisal. Muscle Nerve. 2007;36(4):424-435

3. Koenig M, Monaco AP, Kunkel LM. The complete sequence of dystrophin predicts a rod-shaped cytoskeletal protein. Cell. 1988;53(2):219-228

4. Hoffman EP, Monaco AP, Feener CC, Kunkel LM. Conservation of the Duchenne muscular dystrophy gene in mice and humans. Science. 1987;238(4825):347-350.

5. Angelini C, Peterle E. Old and new therapeutic developments in steroid treatment in Duchenne muscular dystrophy. Acta Myol. 2012;31(1):9-15.

6. Aartsma-Rus A, Krieg AM. FDA approves eteplirsen for Duchenne muscular dystrophy: the next chapter in the eteplirsen saga Nucleic Acid Ther. 2017;27(1):1-3.

7. Finkel RS, et al. Phase 2 a study of ataluren-mediated dystrophin production in patients with nonsense mutation Duchenne muscular dystrophy. PLoS ONE. 2013;8(12):e81302.

8. Chamberlain JR, Chamberlain JS. Progress toward gene therapy for Duchenne muscular dystrophy. Mol Ther. 2017;25(5):1125-1131.

9. Meregalli M, et al. Perspectives of stem cell therapy in Duchenne muscular dystrophy. FEBS J. 2013;280(17):4251-4262.

10. McGreevy JW, Hakim CH, McIntosh MA, Duan D. Animal models of Duchenne muscular dystrophy: from basic mechanisms to gene therapy. Dis Model Mech. 2015;8(3):195-213.

11. Szabo M, Svensson Akusjärvi S, Saxena A, Liu J, Chandrasekar G, Kitambi SS. Cell and small animal models for phenotypic drug discovery. Drug Des Devel Ther. 2017;11:1957-1967.

12. Giacomotto J, et al. Evaluation of the therapeutic potential of carbonic anhydrase inhibitors in two animal models of dystrophin deficient muscular dystrophy. Hum Mol Genet. 2009;18(21):4089-4101.

13. Kawahara G, Kunkel LM. Zebrafish based small molecule screens for novel DMD drugs. Drug Discov Today Technol. 2013;10(1):e91-96.

14. Collins CA, Morgan JE. Duchenne's muscular dystrophy: animal models used to investigate pathogenesis and develop therapeutic strategies. Int J Exp Pathol. 2003;84(4):165-172.

15. Choi IY, et al. Concordant but varied phenotypes among Duchenne muscular dystrophy patient-specific myoblasts derived using a human iPSC-based model. Cell Rep. 2016;15(10):2301-2312.

16. Chal J, et al. Differentiation of pluripotent stem cells to muscle fiber to model Duchenne muscular dystrophy. Nat Biotechnol. 2015;33(9):962-969 
17. Blau HM, Webster C, Pavlath GK. Defective myoblasts identified in Duchenne muscular dystrophy. Proc Natl Acad Sci U S A. 1983;80(15):4856-4860.

18. Jasmin G, Tautu C, Vanasse M, Brochu P, Simoneau R. Impaired muscle differentiation in explant cultures of Duchenne muscular dystrophy. Lab Invest. 1984;50(2):197-207.

19. Delaporte C, Dehaupas M, Fardeau M. Comparison between the growth pattern of cell cultures from normal and Duchenne dystrophy muscle. J Neurol Sci. 1984;64(2):149-160.

20. Barton-Davis ER, Cordier L, Shoturma DI, Leland SE, Sweeney HL. Aminoglycoside antibiotics restore dystrophin function to skeletal muscles of $m d x$ mice. J Clin Invest. 1999;104(4):375-381.

21. Malik V, et al. Gentamicin-induced readthrough of stop codons in Duchenne muscular dystrophy. Ann Neurol. 2010;67(6):771-780

22. Schiaffino S, Rossi AC, Smerdu V, Leinwand LA, Reggiani C. Developmental myosins: expression patterns and functional sig nificance. Skelet Muscle. 2015;5(22).

23. Chong CR, Chen X, Shi L, Liu JO, Sullivan DJ. A clinical drug library screen identifies astemizole as an antimalarial agent. Nat Chem Biol. 2006;2(8):415-416.

24. Dorchies OM, et al. The anticancer drug tamoxifen counteracts the pathology in a mouse model of duchenne muscular dystrophy. Am J Pathol. 2013;182(2):485-504.

25. Lester D. The deterrent effect of executions on homicide. Psychol Rep. 1989;64(1):306.

26. Owens J, Moreira K, Bain G. Characterization of primary human skeletal muscle cells from multiple commercial sources. In Vitro Cell Dev Biol Anim. 2013;49(9):695-705.

27. Lin B, et al. Modeling and study of the mechanism of dilated cardiomyopathy using induced pluripotent stem cells derived from individuals with Duchenne muscular dystrophy. Dis Model Mech. 2015;8(5):457-466.

28. Garrido C, Galluzzi L, Brunet M, Puig PE, Didelot C, Kroemer G. Mechanisms of cytochrome c release from mitochondria. Cell Death Differ. 2006;13(9):1423-1433.

29. Rahimov F, et al. Gene expression profiling of skeletal muscles treated with a soluble activin type IIB receptor. Physiol Genomics. 2011;43(8):398-407.

30. Ge Y, Waldemer RJ, Nalluri R, Nuzzi PD, Chen J. Flt3L is a novel regulator of skeletal myogenesis. J Cell Sci. 2013;126(pt 15):3370-3379.

31. E1 Fakih R, Rasheed W, Hawsawi Y, Alsermani M, Hassanein M. Targeting FLT3 mutations in acute myeloid leukemia. Cells. 2018;7(1):E4.

32. Araki H, et al. Analysis of PPARalpha-dependent and PPARalpha-independent transcript regulation following fenofibrate treatment of human endothelial cells. Angiogenesis. 2009;12(3):221-229.

33. Derynck R, Zhang YE. Smad-dependent and Smad-independent pathways in TGF-beta family signalling. Nature. 2003;425(6958):577-584.

34. Delaney K, Kasprzycka P, Ciemerych MA, Zimowska M. The role of TGF-beta1 during skeletal muscle regeneration. Cell Biol Int. 2017;41(7):706-715.

35. Beastrom N, et al. $m d x^{5} c v$ mice manifest more severe muscle dysfunction and diaphragm force deficits than do $m d x$ mice. $A m J$ Pathol. 2011;179(5):2464-2474.

36. Bulfield G, Siller WG, Wight PA, Moore KJ. X chromosome-linked muscular dystrophy (mdx) in the mouse. Proc Natl Acad Sci U S A. 1984;81(4):1189-1192.

37. Pessina $\mathrm{P}$, et al. Novel and optimized strategies for inducing fibrosis in vivo: focus on Duchenne muscular dystrophy. Skelet Muscle. $2014 ; 4(7)$

38. Blat Y, Blat S. Drug discovery of therapies for Duchenne muscular dystrophy. J Biomol Screen. 2015;20(10):1189-1203.

39. Salani S, Donadoni C, Rizzo F, Bresolin N, Comi GP, Corti S. Generation of skeletal muscle cells from embryonic and induced pluripotent stem cells as an in vitro model and for therapy of muscular dystrophies. J Cell Mol Med. 2012;16(7):1353-1364

40. Hicks MR, et al. ERBB3 and NGFR mark a distinct skeletal muscle progenitor cell in human development and hPSCs. Nat Cell Biol. 2018;20(1):46-57.

41. Sotiropoulos A, et al. Growth hormone promotes skeletal muscle cell fusion independent of insulin-like growth factor 1 up-regulation. Proc Natl Acad Sci USA. 2006;103(19):7315-7320.

42. González MN, et al. HGF potentiates extracellular matrix-driven migration of human myoblasts: involvement of matrix metalloproteinases and MAPK/ERK pathway. Skelet Muscle. 2017;7(1):20.

43. Leung KW, Wong AS. Pharmacology of ginsenosides: a literature review. Chin Med. 2010;5(20).

44. Kim JH, Yi YS, Kim MY, Cho JY. Role of ginsenosides, the main active components of Panax ginseng, in inflammatory responses and diseases. J Ginseng Res. 2017;41(4):435-443.

45. Wang Y, et al. Ginsenoside Rd attenuates myocardial ischemia/reperfusion injury via Akt/GSK-3 $\beta$ signaling and inhibition of the mitochondria-dependent apoptotic pathway. PLoS ONE. 2013;8(8):e70956.

46. Takahashi S. Downstream molecular pathways of FLT3 in the pathogenesis of acute myeloid leukemia: biology and therapeutic implications. J Hematol Oncol. 2011;4(13)

47. Grafone T, Palmisano M, Nicci C, Storti S. An overview on the role of FLT3-tyrosine kinase receptor in acute myeloid leukemia: biology and treatment. Oncol Rev. 2012;6(1):e8.

48. Gredinger E, Gerber AN, Tamir Y, Tapscott SJ, Bengal E. Mitogen-activated protein kinase pathway is involved in the differentiation of muscle cells. J Biol Chem. 1998;273(17):10436-10444.

49. Wu Z, et al. p38 and extracellular signal-regulated kinases regulate the myogenic program at multiple steps. Mol Cell Biol. 2000;20(11):3951-3964

50. Staels B, Dallongeville J, Auwerx J, Schoonjans K, Leitersdorf E, Fruchart JC. Mechanism of action of fibrates on lipid and lipoprotein metabolism. Circulation. 1998;98(19):2088-2093.

51. Najib J. Fenofibrate in the treatment of dyslipidemia: a review of the data as they relate to the new suprabioavailable tablet formulation. Clin Ther. 2002;24(12):2022-2050.

52. Goncalves MD, et al. Fenofibrate prevents skeletal muscle loss in mice with lung cancer. Proc Natl Acad Sci US A. 2018;115(4):E743-E752. 
53. Castillero E, et al. Fenofibrate, a PPAR \{alpha\} agonist, decreases atrogenes and myostatin expression and improves arthritisinduced skeletal muscle atrophy. Am J Physiol Endocrinol Metab. 2011;300(5):E790-E799.

54. MacDonald EM, Cohn RD. TGF $\beta$ signaling: its role in fibrosis formation and myopathies. Curr Opin Rheumatol. 2012;24(6):628-634.

55. Clark RA, McCoy GA, Folkvord JM, McPherson JM. TGF-beta 1 stimulates cultured human fibroblasts to proliferate and produce tissue-like fibroplasia: a fibronectin matrix-dependent event. J Cell Physiol. 1997;170(1):69-80.

56. Magrath P, Maforo N, Renella P, Nelson SF, Halnon N, Ennis DB. Cardiac MRI biomarkers for Duchenne muscular dystrophy. Biomark Med. 2018;12(11):1271-1289.

57. Chang NW, et al. Fenofibrate exhibits a high potential to suppress the formation of squamous cell carcinoma in an oral-specific 4-nitroquinoline 1-oxide/arecoline mouse model. Biochim Biophys Acta. 2011;1812(4):558-564.

58. Ye R, Kong X, Yang Q, Zhang Y, Han J, Zhao G. Ginsenoside Rd attenuates redox imbalance and improves stroke outcome after focal cerebral ischemia in aged mice. Neuropharmacology. 2011;61(4):815-824.

59. Hill CS, Duran S, Lin ZX, Weber K, Holtzer H. Titin and myosin, but not desmin, are linked during myofibrillogenesis in postmitotic mononucleated myoblasts. J Cell Biol. 1986;103(6 pt 1):2185-2196.

60. Li Z, Marchand P, Humbert J, Babinet C, Paulin D. Desmin sequence elements regulating skeletal muscle-specific expression in transgenic mice. Development. 1993;117(3):947-959.

61. Sun C, et al. Common and distinctive functions of the hippo effectors taz and yap in skeletal muscle stem cell function. Stem Cells. 2017;35(8):1958-1972.

62. Pratt SJP, Lawlor MW, Shah SB, Lovering RM. An in vivo rodent model of contraction-induced injury in the quadriceps muscle. Injury. 2012;43(6):788-793.

63. Stone MR, et al. Absence of keratin 19 in mice causes skeletal myopathy with mitochondrial and sarcolemmal reorganization. J Cell Sci. 2007;120(pt 22):3999-4008.

64. Lovering RM, De Deyne PG. Contractile function, sarcolemma integrity, and the loss of dystrophin after skeletal muscle eccentric contraction-induced injury. Am J Physiol, Cell Physiol. 2004;286(2):C230-C238.

65. Tohyama S, et al. Distinct metabolic flow enables large-scale purification of mouse and human pluripotent stem cell-derived cardiomyocytes. Cell Stem Cell. 2013;12(1):127-137. 